\title{
PID performance of the ALICE-TOF detector in Run 2
}

\author{
Nicolò Jacazio*t \\ University of Bologna and INFN \\ E-mail: nicolo.jacaziodbo.infn.it
}

In these proceedings we report on the status of the ALICE Time-Of-Flight (TOF) detector. The running performance of the Run 1 (2009-2013) and Run 2 (2015-present) data taking campaigns are compared. The particle identification capabilities of the detector are presented and discussed in the light of the improved detector calibration that allowed to reach a timing resolution of $56 \mathrm{ps}$.

Sixth Annual Conference on Large Hadron Collider Physics (LHCP2018)

4-9 June 2018

Bologna, Italy

* Speaker.

${ }^{\dagger}$ For the ALICE Collaboration 


\section{Introduction}

The ALICE experiment $[1,2]$ is one of the four main experiments recording collisions at the CERN Large Hadron Collider. One main point of its physics program is the study of the dense and hot matter created in ultra-relativistic heavy-ion collisions i.e. the so called Quark-Gluon-Plasma. The ALICE experiment is not limited to the study of heavy-ion collisions but it extends to smaller systems such as $\mathrm{pp}$ and $\mathrm{p}-\mathrm{Pb}$ collisions. These cover interesting physics cases on themselves but are also be used as a useful reference for the heavy-ion case. Identifying the particles produced in the collisions is fundamental in many analyses. This is the reason why the ALICE detector is capable of providing Particle IDentification (PID) over a continuous interval of momentum [3]. The ALICE detector was designed to have good tracking capabilities down to very low $p_{\mathrm{T}}(\sim 100 \mathrm{MeV} / c)$. In order to achieve this performance it uses a moderate magnetic field in the mid-rapidity region $(0.2$ or $0.5 \mathrm{~T}$ ) coupled with a very low material budget. The high density of charged particles created in a single heavy-ion collision requires high granularity detectors. The tracking is performed in the region close to the collision vertex with a Silicon detector (Inner Tracking System or ITS) and then continued in a Time Projection Chamber (TPC) towards the outer layers of the detector. Both detectors provide an analogic measurement of the particle energy loss and this information allows to differentiate between different particle species for what concerns low momenta (below $\sim 800$ $\mathrm{MeV} / \mathrm{c}$ ). The Time-Of-Flight (TOF) [4, 5] is used to complement these detectors and extend the momentum reach of the PID for charged particles.

\section{The ALICE Time-Of-Flight detector and its PID capabilities}

The TOF detector covers a large area $\left(\sim 141 \mathrm{~m}^{2}\right)$ with cylindrical geometry with internal radius $\sim 3.7 \mathrm{~m}$ from the beam axis. With respect to the interaction point the TOF system uniformly covers a pseudorapidity $(\eta)$ region of 0.9 with axial symmetry, for a total coverage of 1.8 units of $\eta$ in the full azimuthal angle. The sensitive unit of the TOF detector is based on the Multigap Resistive Plate Chamber (MRPC) technology, built from two stacks of five single gas gaps. The whole detector is segmented into 1593 MRPC strips, each one covering $120 \times 7.4 \mathrm{~cm}^{2}$ and mounted inside 87 separate modules which are grouped into 18 sectors covering the whole $2 \pi$ angle. To achieve the high granularity needed to guarantee a low occupancy even at the high charged particle densities of $\mathrm{Pb}-\mathrm{Pb}$ collisions, the single MRPC strip is segmented into two rows of 48 pickup pads of $3.5 \times 2.5 \mathrm{~cm}^{2}$, accounting for 96 readout pads per strip and 152928 total readout channels. In addition to its PID capabilities, the TOF system provides a dedicated trigger for cosmic rays. In all its years of operation the TOF detector proved itself to be very reliable: an example can be seen in Fig. 1a where the trigger rate for cosmic events is shown as a function of time and it is found to be stable, indicating no sign of detector degradation so far. The same conclusion can be drawn from the measurement of the current passing trough all 1593 MRPCs. In Fig. 1c the current without any beam circulating in the LHC is plotted as a function of time and it is found to be very stable with the exception of few points measured just after the finalization of the detector installation and due to the initial MRPCs HV conditioning. The stability of the current and its low value indicate that the detector has low noise and no signs of ageing is observed from 2009 till 2017. The current is also shown as a function of the instantaneous luminosity in Fig. 1b. The 
stability and the linearity of the response without any sign of saturation make the TOF detector a good candidate for a luminometer. The ALICE TOF MRPC was designed to ensure large particle

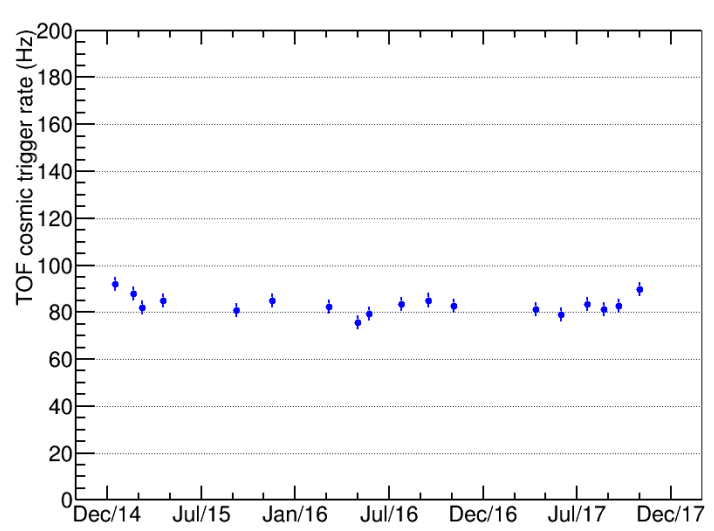

(a)

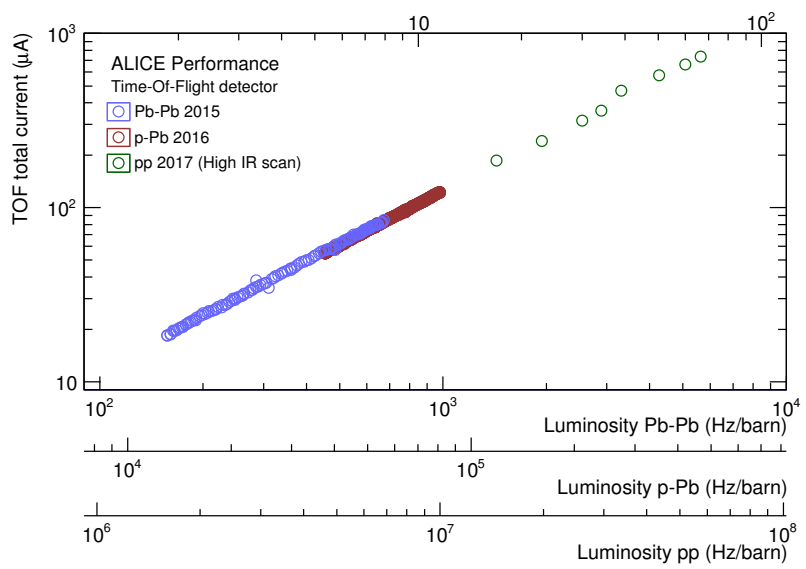

(b)

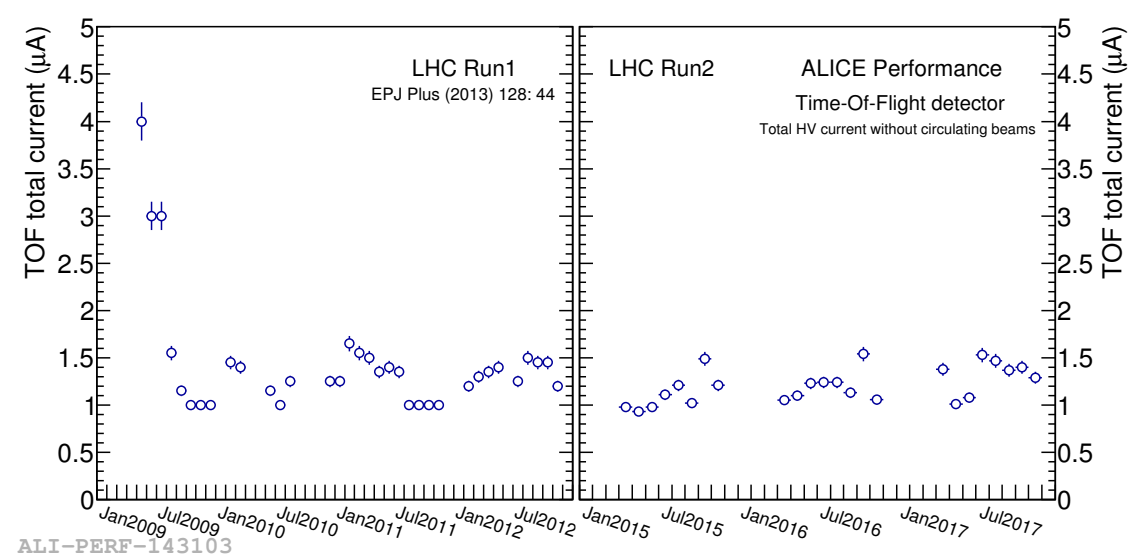

(c)

Figure 1: Summary of the running conditions of TOF during Run 1 and Run 2. In (a) the rate of the cosmic events triggered by the TOF detector is shown as a function of time. In (b) and (c) the total current passing trough the TOF MRPCs is shown as a function of the instantaneous luminosity (with beam) and as a function of time (without beam) respectively.

detection efficiencies $(\sim 99 \%)$, nonetheless this efficiency has to be coupled with the detector geometrical acceptance and the global tracking algorithm of the ALICE experiment, resulting in a lower effective efficiency shown in Fig. 2 for $\mathrm{p}-\mathrm{Pb}$ collisions as measured from Run 1 and Run 2 data. The discrepancies between the two periods can be understood as follows: during the Long Shutdown 1 phase ALICE finished the installation of all the modules of the TRD detector which is located between TPC and TOF, thus adding more material for particles to cross. These changes are the cause for the difference in the overall track matching efficiencies, indicating again no sign of ageing of the MRPCs. The data collected in the first months after the start of Run 2 was used to extract an improved calibration. Among the several technical changes used for the calibration it is 


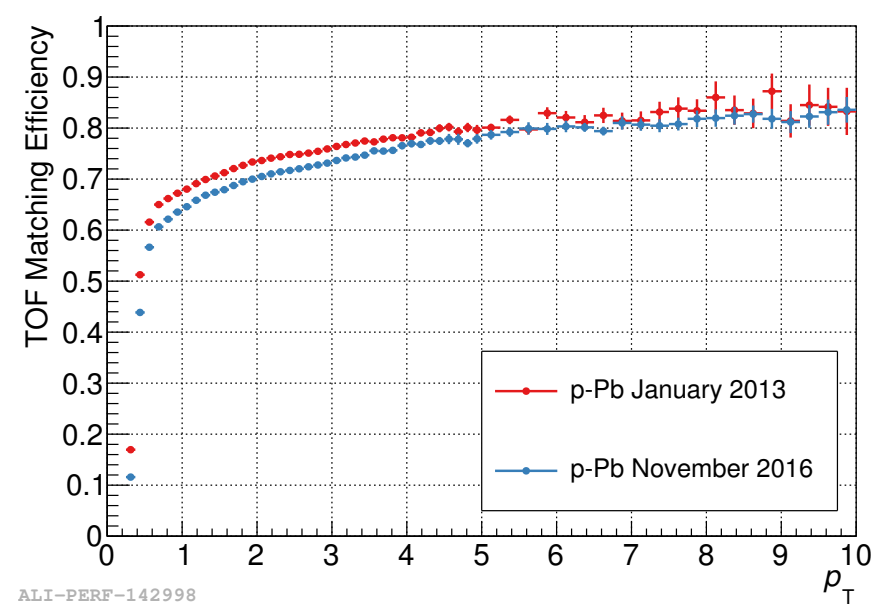

Figure 2: Overall track matching efficiency as measured in $\mathrm{p}-\mathrm{Pb}$ at $\sqrt{s_{N N}}=5.02 \mathrm{TeV}$ collisions during Run 1 and Run 2. The difference of the two efficiencies is due to the installation of all TRD modules.

important to mention the single channel granularity (in the past 8-channel groups were used) and the use of a finer point by point parameterization of the correction as a function of the measured Time-Over-Threshold (in the past limited to a $5^{\text {th }}$ order polynomial). Both standard and improved calibration curves are shown for one channel in Fig. 3a. The effect of the calibration on a single channel is shown in Fig. $3 \mathrm{~b}$ and during the whole 2017 the new procedure proved to be very stable as $88 \%$ of all TOF channels showed RMS lower than 20 ps. The Time-Of-Flight measurement can simply be obtained by subtracting the event collision time $\left(t_{\text {event }}\right)$ from the time measured with the TOF detector $\left(t_{T O F}\right)$, as reported in Eq. 2.1; the resolution on the discriminating quantity $T O F-t_{\text {exp }}$ is defined in Eq. 2.2 where $\sigma_{\text {tracking }}$ identifies the effect of tracking on $t_{\text {exp }}$ i.e. the expected particle Time-Of-Flight. The components that contribute to the uncertainty on $t_{T O F}$ and $t_{\text {event }}$ are listed in Eq. 2.3 and Eq. 2.4.

$$
\begin{aligned}
& \text { Time-Of-Flight } \equiv T O F=t_{T O F}-t_{\text {event }} \\
& \sigma_{T O F-t_{\text {exp }}}=\sigma_{t_{T O F}}^{2}+\sigma_{\text {tracking }}^{2}+\sigma_{t_{\text {event }}}^{2} \\
& \sigma_{t_{T O F}}^{2}=\sigma_{M R P C}^{2}+\sigma_{T D C}^{2}+\sigma_{F E E}^{2}+\sigma_{\text {Calibration }}^{2} \\
& \sigma_{t_{\text {event }}} \sim \frac{\sigma_{t_{T O F}}}{\sqrt{n_{\text {tracks }}}}
\end{aligned}
$$

The resolution on the simple time measurement $\left(\sigma_{t_{T O F}}\right)$ depends mostly on fixed elements which are intrinsic of the detector such as the MRPC resolution $\left(\sigma_{M R P C}\right)$ and the one given by electronic readout chain ( $\sigma_{T D C}$ and $\sigma_{F E E}$ ). Thanks to the improvements described above the contribution due to the calibration $\left(\sigma_{\text {Calibration }}\right.$ ) was significantly reduced and allowed to achieve resolution of $\sim 56 \mathrm{ps}$, as reported in Fig. 4a. The overall effect on the separation power is depicted in Fig. 4b. The determination of the event collision time needed to compute the Time-Of-Flight can be performed autonomously by the TOF detector itself with the iterative procedure described in [6]. Thanks to this method the measurement of the Time-Of-Flight can benefit further from the improved precision on $t_{T O F}$ as reported in Eq. 2.4. The $\sigma_{t_{\text {event }}}$ depends on the number of tracks available for the 


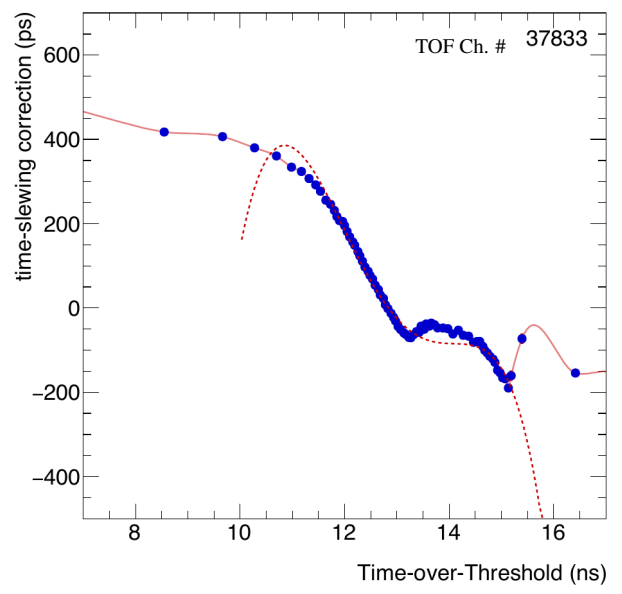

(a)

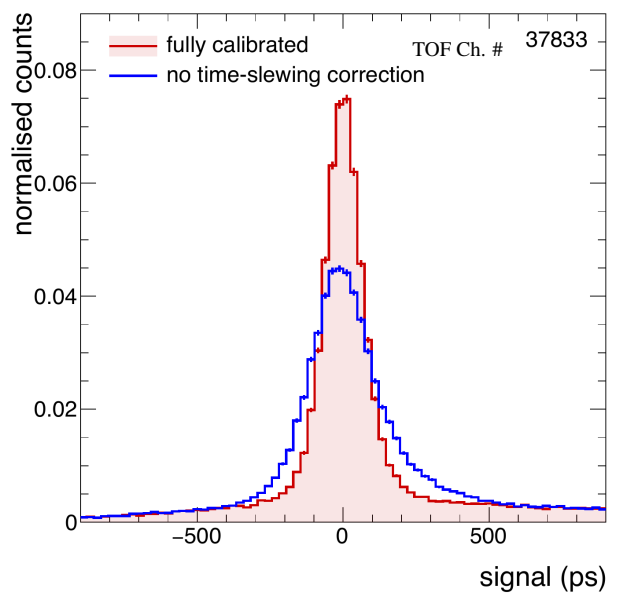

(b)

Figure 3: Summary of the status for the improved calibration procedure. In (a) the old (dashed line) and new (solid line) parameterization for the time slewing correction are drawn. In (b) the time signal distribution for a particular channel is shown before and after the time slewing correction is applied.

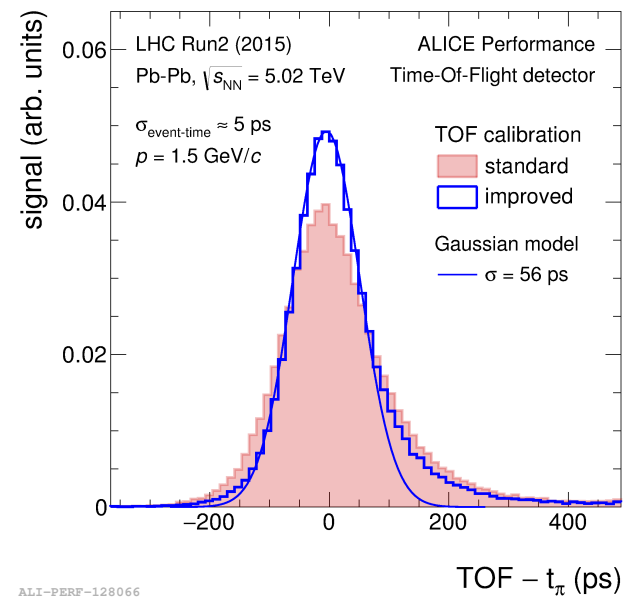

(a)

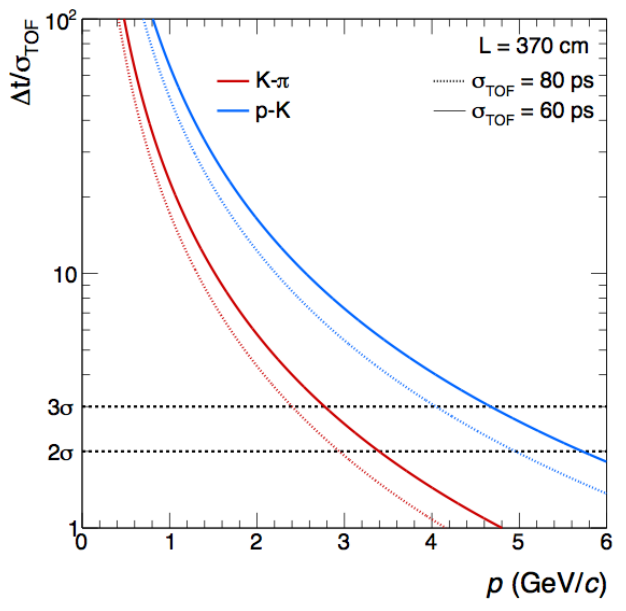

(b)

Figure 4: (a) Signal distribution with resolution as measured by the full ALICE TOF detector during Run 2. (b) the impact of the improved resolution on the PID is depicted together with lines at 2 and 3 sigma.

$t_{\text {event }}$ evaluation ( $\left.n_{\text {tracks }}\right)$. The improvement between Run 1 and Run 2 of the event collision time precision is shown in Fig. 5a. With the improved calibration the resolution is found to be better than 5 ps for events with more than $\sim 600$ tracks matched to TOF (Fig. $5 b$ ). All the developments and procedures described above are summarized in Fig. 6 where the $\beta$ of each particle is shown as a function of its momentum as measured in $\mathrm{Pb}-\mathrm{Pb}$ collision at $\sqrt{s_{N N}}=5.02 \mathrm{TeV}$. The momentum slices of the $\beta$ distribution are shown in Fig. 7, where it is possible to appreciate the separation of the signals of each particle species. Starting at $400 \mathrm{MeV} / \mathrm{c}$ in Fig. $7 \mathrm{a}$ it is possible to identify the 
signals of pions, muons and electrons. At larger momenta (Fig. 7b) the peaks of protons and kaons are visible, while light nuclei can also be identified starting from $\sim 3 \mathrm{GeV} / \mathrm{c}$ (Fig. 7c). As expected from Fig. $4 \mathrm{~b}$ the proton and kaon peaks are still separated at $5 \mathrm{GeV} / c$ (Fig. 7d).

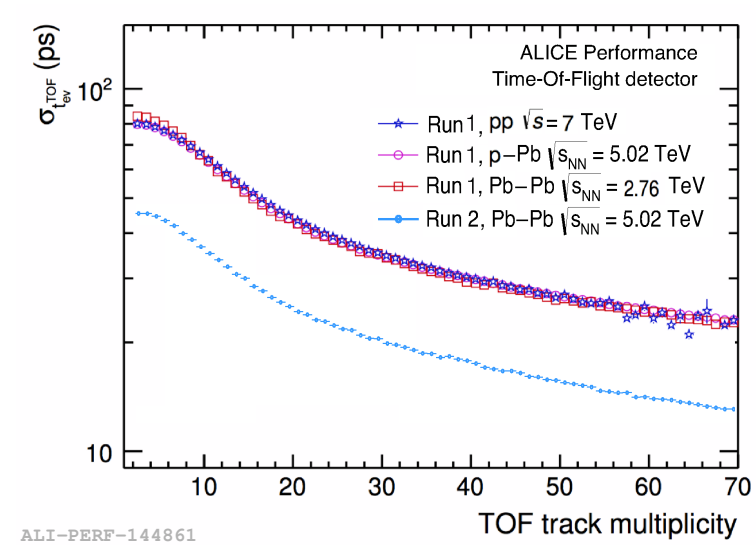

(a)

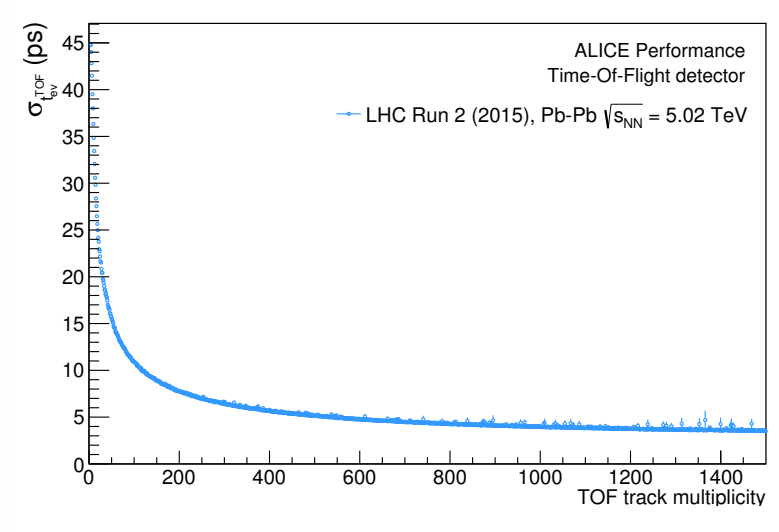

(b)

Figure 5: Collision event collision time resolution as measured with the TOF detector in both Run 1 and Run 2 as a function of the number of reconstructed tracks with a time measurement. Two ranges are proposed: in (a) the event collision time resolution is shown up to multiplicities of 70 and compared across multiple collision systems, in (b) the multiplicity range is enlarged so as to fit the values reached in heavy-ion collisions.

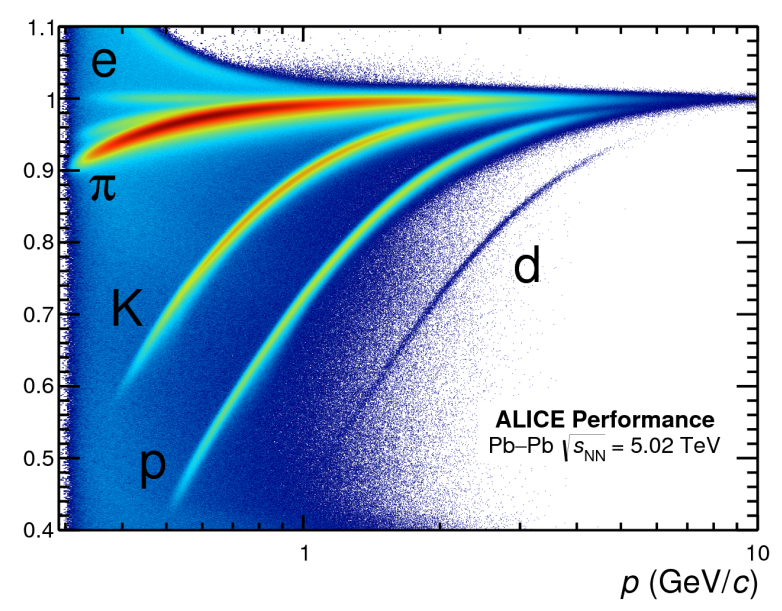

Figure 6: Particle velocity $(\beta)$ distribution as a function of momentum for tracks reconstructed with the ALICE experiment in $\mathrm{Pb}-\mathrm{Pb}$ collisions at $\sqrt{s_{N N}}=5.02 \mathrm{TeV}$.

\section{Conclusions}

The working performance of the ALICE Time-Of-Flight detector was discussed and it was shown that the detector does not appear to suffer from ageing in any way. This information is crucial as the TOF detector will continue the data taking also after Run 2 with limited modifications to the readout chain. During Run 2 an improvement in the TOF calibration made it possible to reach 
timing resolutions of $\sim 56 \mathrm{ps}$, thus improving significantly the Particle IDentification capabilities and extending its reach. In the light of these new developments the PID performance now allows an improved separation of electrons, muons, pions, kaons, protons and light nuclei over a wide range of momenta.

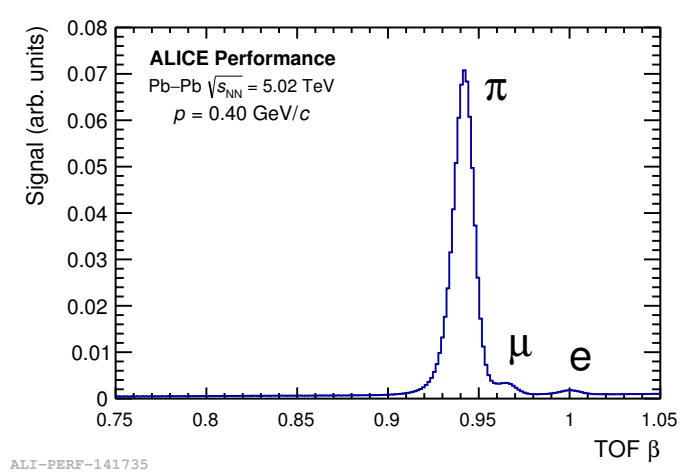

(a)

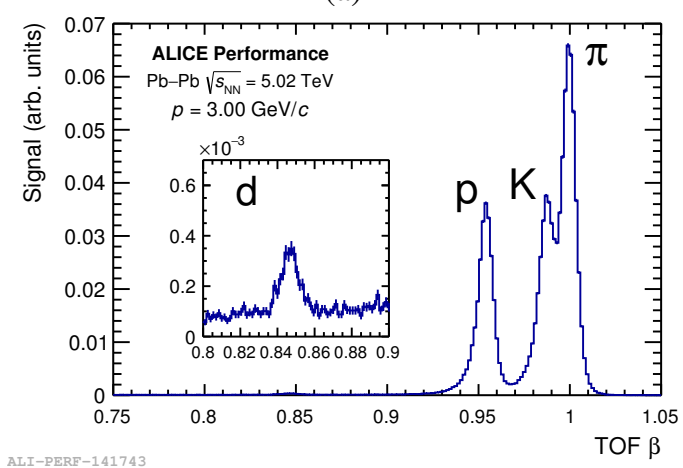

(c)

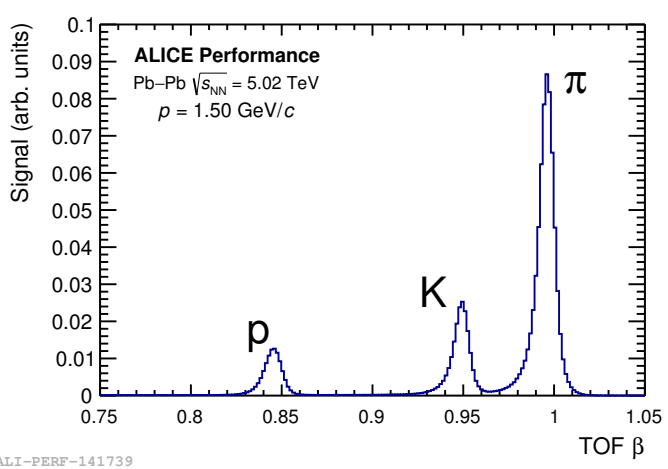

(b)

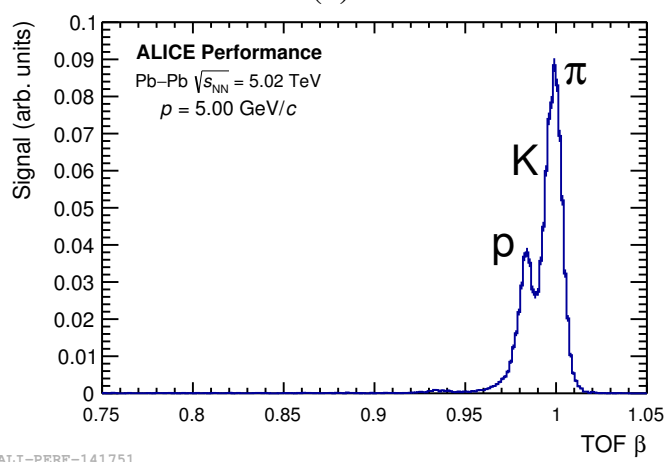

(d)

Figure 7: Distribution of the particle velocity $(\beta)$ as measured with the TOF detector in different momentum intervals. The signals from each particle species are clearly visible up to high momenta.

\section{References}

[1] ALICE collaboration, K. Aamodt et al., The ALICE experiment at the CERN LHC, JINST 3 (2008) S08002.

[2] ALICE collaboration, B. B. Abelev et al., Performance of the ALICE Experiment at the CERN LHC, Int. J. Mod. Phys. A29 (2014) 1430044 [1 402.4476$].$

[3] ALICE collaboration, J. Adam et al., Particle identification in ALICE: a Bayesian approach, Eur. Phys. J. Plus 131 (2016) 168 [1602.01392].

[4] ALICE collaboration, G. Dellacasa et al., ALICE technical design report of the time-of-flight system (TOF), CERN-LHCC-2000-012 (2000) .

[5] ALICE collaboration, P. Cortese et al., ALICE: Addendum to the technical design report of the time of flight system (TOF), CERN-LHCC-2002-016 (2002) .

[6] ALICE collaboration, J. Adam et al., Determination of the event collision time with the ALICE detector at the LHC, Eur. Phys. J. Plus 132 (2017) 99 [1610 . 03055]. 